\title{
Extracorporeal Membrane Oxygenation and Prone Position Ventilation on Critically ill COVID-19: A Case Report and Literature Review
}

Jingchen Zhang

Zhejiang University

Xujian He

Zhejiang University

Jia Hu

Zhejiang University

Chenkan Chen

Zhejiang University

Xuesheng Ye

Zhejiang University

Tong Li ( $\nabla$ drli@zju.edu.cn )

Zhejiang University https://orcid.org/0000-0003-2011-1894

\section{Case report}

Keywords: COVID-19, prone position ventilation, mechanical ventilation, awake ECMO

Posted Date: June 5th, 2020

DOl: https://doi.org/10.21203/rs.3.rs-32686/v1

License: (9) (i) This work is licensed under a Creative Commons Attribution 4.0 International License. Read Full License 


\section{Abstract}

Background: COVID-19 broke out all over the world, and the mortality rate is extremely high. We report the successful experience of a critically ill COVID-19 patient who underwent long-term extracorporeal membrane oxygenation (ECMO) and multiple prone position ventilation (PPV) treatments.

Case presentation: A 53-year-old male patient was sent to our hospital after 11 days of cough and 9 days of fever. According to his CT scan and real-time reverse transcription-polymerase chain reaction assay to throat swap, his nucleic acid was positive, confirming that he was infected with COVID-19. Subsequently, he was sent to ICU for respiratory failure. Afterwards, the patient received antiviral drug, tiny amount of glucocorticoid, and respiratory support, including mechanical ventilation, but the affect was poor. In the 28th day of his admission, veno-venous ECMO and PPV were used, combining with awake ECMO and other comprehensive rehabilitation. In the 17th day of ECMO, the patient started getting better and his chest CT and lung compliance improved. The ECMO was removed in the 27th days, after which the mechanical ventilation was gotten rid of in the 9th day. Then he was transferred to rehabilitation department.

Conclusions: COVID-19 can damage lung tissues and cause evident inflammatory exudation, affecting oxygenation function. It is effective to use PPV, awake ECMO, and comprehensive rehabilitation to cure patients with critical COVID-19 and respiratory failure. Our experience hopes to be promoted to play a positive role.

\section{Background}

Coronavirus Disease 2019 (COVID-19) was caused by SARS-CoV-2,a novel coronavirus. The first case was confirmed in China, but COVID-19 has become a worldwide epidemic. SARS-CoV2 virus infection leads to excessive exudation of pulmonary capillaries, manifesting interstitial edema, and ground-glass changes can be seen in the image ${ }^{1}$. COVID-19 has various clinical manifestations, including fever and respiratory track syndromes. Some COVID-19 patients experience mild symptoms, and there is no obvious abnormity in chest $\mathrm{CT}$, while a few patients get sever respiratory failure quakily, with high death rate. As for the latter patients, ventilator or Extracorporeal membrane oxygenation (ECMO) is needed to support breathing ${ }^{2}$.

ECMO is a kind of technology that can provide gas exchange and support cardiac function for patients with ARDS or cardiac failure. After the trial of CESAR in 2009,veno-venous ECMO was established and widely used for the treatment of severe patients with ARDS ${ }^{3}$. However, COVID-19 need a relatively longer course of treatment than other known viral pneumonia. At the same time, it could progress rapidly, contributing to respiratory failure ${ }^{4}$. Refractory hyoxemia marks the most common clinical manifestation in critically ill patients with COVID-19. Previous studies proved that prone position ventilation (PPV) could reduce the death rate of patients with severe ARDS. According to some reports, the combination of ECMO 
and PPV can effectively cure viral pneumonia ${ }^{5}$. From the studies on its complications, it was discovered that the combination is safe ${ }^{6}$.

The prevalence of ARDS for patients with COVID-19 is associated with extremely high mortality, especially before the introduction of effective antiviral treatment ${ }^{7}$. A clinical case was presented, in which the ARDS patient suffering from COVID-19 survived after the application of ECMO.

\section{Case Report}

A 53-year-old man was admitted to our hospital, experiencing 11 days of cough, sputum, and 9 days of fever. His chest X-ray indicated ground-glass opacitie appears in the lower of right lung (Fig. 1A). SARSCoV-2 was confirmed by the real-time reverse transcription-polymerase chain reaction (RT-PCR) assay and the nasopharyngeal specimen was positive. The patient was given NFHC, Arbidol, Lopinavir + Ritonavir, and Methylprednisolone (40 mg/ $\left.{ }^{\star} 8 \mathrm{~d}\right)$.

After 12 days of treatment, he developed respiratory failure, with partial pressure of oxygen/ fraction of inspiration $\mathrm{O}_{2}(\mathrm{PaO} 2 / \mathrm{FiO} 2)<150 \mathrm{mmHg}$ for 12 hours and respiratory rate $>30 /$ min over 6 hours, showing that the patient satisfied the sign of mechanical ventilation and was admitted to intensive care unit (ICU) for treatment. A chest X-ray showed that the density of lower lungs lobe increased, with spread plaque image and blur border (Fig. 1B). Over the next 96 hours, lung protective strategies and a restrictive fluid strategy were applied, but the patient got worse, with terrible lung compliance, hypoxemia, as well as the dense opacification of diffuse lesion in lungs (Fig. 1C). PPV was adopted, together with sedative and analgesic drugs, muscle relaxant, lung recruitment and phlegm drainage, but there was no significant improvement for the patient.

After 12 days of mechanical ventilation, the tracheotomy was performed for the patient, but his virus was still positive by detecting. The patient's $\mathrm{PaO}_{2} / \mathrm{FiO}_{2}$ dropped to 80 and lasted for $24 \mathrm{~h}$, which conformed to the implementing standard of ECMO. The patient was given ECMO treatment in the 16th day of mechanical ventilation. In terms of the initiation parameter of ECMO, the mode is $\mathrm{V}-\mathrm{V}$, the location of intubation is in the left femoral vein and right internal jugular vein, and the size of tube is $16 \mathrm{f}$ and $22 \mathrm{f}$. A chest $\mathrm{X}$-ray showed that the catheter was in a reasonable position and the pulmonary lesions were aggravated (Fig. 1D).

During running time, activated partial thromboplastin time(APTT)was maintained $40-60 \mathrm{sec}$. The ECMO system was set to blood flow of 3.5-4.0 L/min and a sweep flow rate of 4.0-7.0 L/min, which was adjusted according to the $\mathrm{PaCO}_{2}$ obtained by blood gas analysis. The target coagulation profile was monitored by detecting the levels of APTT. During ECMO therapy, the target oxygenation was a normal $\mathrm{PaCO}_{2}$ and $\mathrm{PaO}_{2}$.

With the support of ECMO, haemolysis occurred, with plasma-free hemoglobin $\nabla 120 \mathrm{mg} / \mathrm{dL}$. In addition, enterococcus faecalis led to infectious shock and unstable blood volume, but there was no any 
improvement after the adequate fluid resuscitation. ECMO catheter was replaced with right femoral vein and left internal jugular vein and the size of tubes was $19 \mathrm{f}$ or $22 \mathrm{f}$. Then the blood flow of ECMO got better, and haemolysis and infectious shock were controlled.

The re-examination of CT illustrated lesions in both lungs and severe exudation (Fig. 2, 1A-2A). At the early stage of ECMO, PPV was also carried out at the same time. The specific method of implementation: four nurses helped the patient turn over, one nurse checked the ECMO regularly and respiratory physician was responsible for the management and protection of the intubation. One doctor ensured the stability and flowing of the ECMO tubes, in case of pressure sores, so some materials were placed between skin and bed, and skin and tube to prevent pressure sores and bandage was applied to fix the tube of ECMO.

During the operation of ECMO $(12 \mathrm{~h} / \mathrm{d})$, the ventilator mode setting was BIPAP, $26-30 \mathrm{cmH} 2 \mathrm{O}$ PIP, 6$8 \mathrm{cmH}_{2} \mathrm{O}$ PEEP and 18-25 rpm RR. The parameters of PPV: PEEP was down- regulated $2 \mathrm{cmH} 2 \mathrm{O}$ and $22-$ 26PIP, but other parameters kept steady. Except prone position, other care bundles were also taken, including hand washing, closed endotracheal suction, and appropriate sedation to assist the improvement (Fig. 2,1B-2B). At the 17th day after ECMO initiation, exhaled tidal volumes were increased to $6 \mathrm{~mL} / \mathrm{kg}$ and chest radiographs showed that lung effusion improved, after which the combination of awake ECMO and other comprehensive on bed and floor rehabilitation exercises were adopted.

The patient did the rehabilitation on bed and ground. Bending arms and lowing legs were bed exercises. $\mathrm{He}$ also sat on the side of bed for 30 minutes every day and accepted passive functional exercise for legs. Later, his standing position was also trained. After the patient was sent to ICU, he was given nutrition through jejunal nutrient canal, aiming at $25-30 \mathrm{kcal} / \mathrm{kg}$ and gradually increased the volume of eating food every time. Nucleic acid test of SARS-CoV-2 virus became negative 3 days after ECMO application. In the 27th day of ECMO support, with improved chest CT scan (Fig. 2, 1C-2C), the patient got rid of V-V ECMO. During the period of therapy, the parameters of mechanical ventilator and blood gas analysis were monitored (Fig. 3). In the 9th day of removal, the man was successfully closed casing. After a 4-day high flow nasal cannula oxygen therapy, he was transferred to the rehabilitation department.

\section{Discussion}

At present, initial reports from China and Italy suggest high mortality and stressed ICU capacity ${ }^{8-9}$, with more than $10 \%$ of death rate in some regions. Served as a designated, our center admitted and treated a total of 104 patients, 78 of whom were seriously ill. Reports described that COVID-19 patients who were hospitalized to the ICU and rarely recovered. The patients supported with ECMO was reported less than on week. Although this case patient experienced long-term ECMO treatment, there was no report on it.

The case we studied was an early case of COVID-19 transmission, with rapid progression and poor response to antiviral drugs after the onset of the disease. The oxygenation still got worse in patients after the use of NIV, which further confirmed that COVID-19 has a long course of disease and oxygenation deteriorates rapidly, distinguishing it from traditional viral pneumonia ${ }^{10}$. Some COVID-19 patients may 
require a high-level pressure support of ventilation. However, possessed with high airway pressure, oxygen concentration, severe viral infections, along with protein exudation, mechanical ventilation can lead to severe lung injury and decreased lung compliance ${ }^{11}$.

The treatment for early critical patients is conservative intubation strategy. However, once the disease progresses, with high systemic organ failure and low cure rate, ECMO can be operated to guarantee the situation of oxygenation for patients. But ECMO is invasive operation, with high incidence rate, needing well equipped ECMO center and experienced and skilled medical workers to do it together. In our center, 10 cases of severe COVID-19 patients obtained the treatment of ECMO, 7 of whom were removed the machine successfully. Our comprehensive treatment, with ECMO as bridge, has made great achievements for patients suffering from COVID-19.

PPV can improve the oxygenation for ARDS patients, especially when the position lasts a longtime (17 or $18 \mathrm{~h})^{12}$. Two recent meta-analyses showed that PPV can reduce the death rate for patients in ICU ${ }^{13}$. After the ECMO treatment for oxygenation and stable circulation, this patient was also given PPV. In terms of his $\mathrm{CT}$, the lung lesions distribute along with the gravity and the atrophy of the lung tissue in the back is evident. Out of cure but not to maintain the oxygenation, we performed PPV, the lung CT scan and clinical manifestation indicating that the treatment achieved obvious effect.

The patient tested negative for virus in phlegm and alveolar lavage fluid. In the 17th day, he accepted awake ECMO and his lungs improved a lot. During this process, artificial airway and positive pressure ventilation cannot stop the ventilator-associated pneumonia and ventilator-induced lung injury ${ }^{14}$. In the early stage, for his activity and rehabilitation, sedative and analgesic drugs were reduced to keep the patient conscious. Spontaneous ventilator can promote the even distribution of lung ventilation, and also reduce the occurrence of ventilator-induced diaphragm dysfunction ${ }^{15}$.

Except for the operation of awake ECMO, the patient adopted various rehabilitation methods, including stand by bed and ride a bike, after which his muscle power improved a lot. Awake ECMO allowed the patient to change multiple postures and he was encouraged to cough, which had apparent advantages in phlegm drainage ${ }^{16}$. On the contrary, the COVID-19 patient required a high level of care and protection, which was difficult to implement awake ECMO. It is hard for elderly patients to do rehabilitation exercise for their poor basic living ability, so more health care staff and workers are needed, which is a massive challenge for us.

Multiple complications can develop in the operation of ECMO, and hemorrhage is the common one. In the present case, conservative anticoagulation strategy was carried out, because of the long course of disease, early stage of hemorrhage in digestive track, and high risk of bleeding. Some studied proved that for severe patients, as the use time of ECMO increases, the risk of bleeding will rise at the same time. One of the essential steps is to control the bleeding in the process of ECMO operation ${ }^{17}$. The APTT was set between 40 to $60 \mathrm{~s}$. Integrating our experience, in addition to the combination of awake ECMO and active 
rehabilitation, a low level of anticoagulation scheme is necessary. The patient should be closely monitored in case of the complication of hemorrhage that should be managed in time.

As a bridge to recovery approach, V-V ECMO was quickly introduced to support the patient. The patient was discharged in the 13th day with full recovery, and there were no other adverse effects. Before he left our center, his lung function improved and got rid of oxygen therapy. Other 10 same cases accepted the combination treatment (ECMO and PPV), 7 of whom have can successfully breath without ECMO. Therefore, this method deserves to be promoted.

\section{Conclusion}

Severe COVID-19 patients have high rate of death, especially for old people. Conventional ventilator treatment is not effective for critically ill patients. Although ECMO operation leads to multiple complications, its clinical effect cannot be neglected. For some patients, if the effect of conventional ventilator is terrible, they can try the ECMO treatment.

\section{Declarations}

\section{Ethics approval and consent to participate}

This study was approved by the Human Ethics Committee of the First Affiliated Hospital Zhejiang university. The patient gave consent for publication of this case report and images.

\section{Consent for publication}

Written informed consent was obtained from the patient for publication of this case report and accompanying images.

\section{Availability of data and materials}

The datasets used during the current study are available from the corresponding author on reasonable request.

\section{Competing interests}

The authors have declared no competing interests.

\section{Funding}

None. 


\section{Authors' Contributions}

JC Zhang generated the idea of writing the case report and was the consultant in charge of the patient. JC Zhang and XJ He reviewed the case notes of the patient and wrote the original draft of the case presentation. $\mathrm{J} \mathrm{Hu}, \mathrm{T} \mathrm{Li}, \mathrm{CC}$ Kan and XS Ye significantly revised the original draft and added the conclusions and references. All authors contributed to the final version of the manuscript

\section{Acknowledgments}

The authors would like to thank all participants of the study, the nurses and clinical staff who are providing care for the patients.

\section{References}

1. Xu Z, Shi L, Wang Y, et al. Pathological findings of COVID-19 associated with acute respiratory distress syndrome. The Lancet Respiratory Medicine. 2020;8(4):420-422.

2. Yang $X, Y u Y, X u$ J, et al. Clinical course and outcomes of critically ill patients with SARS-CoV-2 pneumonia in Wuhan, China: a single-centered, retrospective, observational study. The Lancet Respiratory Medicine. 2020.

3. Peek GJ, Mugford M, Tiruvoipati R, et al. Efficacy and economic assessment of conventional ventilatory support versus extracorporeal membrane oxygenation for severe adult respiratory failure (CESAR): a multicentre randomised controlled trial. Lancet 2009; 374: 1351-1363.

4. Bhatraju PK, Ghassemieh BJ, Nichols M, et al. Covid-19 in Critically III Patients in the Seattle Region Case Series. New England Journal of Medicine. 2020.

5. Hraiech S. Prone positioning during veno-venous extracorporeal membrane oxygenation for severe acute respiratory distress syndrome in adults. Minerva Anestesiologica, 2013, 80(3):307-313.

6. Schmidt, Matthieu, Stewart, Claire, Bailey, Michael, et al. Mechanical ventilation management during extracorporeal membrane oxygenation for acute respiratory distress syndrome: a retrospective international multicenter study. critical care medicine, 2015, 43(3):654-664.

7. Guan W-j, Ni Z-y, Hu Y, et al. Clinical Characteristics of Coronavirus Disease 2019 in China. New England Journal of Medicine. 2020;382(18):1708-1720.

8. Grasselli G, Pesenti A, Cecconi M. Critical care utilization for the COVID-19 outbreak in Lombardy, Italy: early experience and forecast during an emergency response. JAMA. 2020.

9. Chen N, Zhou M, Dong X, et al. Epidemiological and clinical characteristics of 99 cases of 2019 novel coronavirus pneumonia in Wuhan, China: a descriptive study. The Lancet. 2020;395(10223):507-513.

10. Arentz M, Yim E, Klaff L, et al. Characteristics and outcomes of 21 critically ill patients with COVID-19 in Washington State. JAMA. 2020. 
11. Borba MGS, Val FFA, Sampaio VS, et al. Effect of high vs low doses of chloroquine diphosphate as adjunctive therapy for patients hospitalized with severe acute respiratory syndrome coronavirus 2 (SARS-CoV-2) infection: a randomized clinical trial. JAMA Netw Open 2020;3(4): 208857.

12. Silversides JA, Ferguson ND. Clinical review: Acute respiratory distress syndrome - clinical ventilator management and adjunct therapy. Crit Care. 2013;17(2):225.

13. Sud S, Friedrich JO, Taccone P, et al. Prone ventilation reduces mortality in patients with acute respiratory failure and severe hypoxemia: systematic review and meta-analysis. Intensive Care Med. 2010;36(4):585-599.

14. Nishimura $\mathrm{M}, \mathrm{Honda} \mathrm{O}$, Tomiyama $\mathrm{N}$, et al. Body position does not influence the location of ventilator induced lung injury. Intensive Care Med, 2000, 26: 1664-1669.

15. Santana MCE, Garcia CSNB, Xisto DG, et al. Prone position prevents regional alveolar hyperinflation and mechanical stress and strain in mild experimental acute lung injury. Respir Physiol Neurobiol, 2009, 167:181-188.

16. Langer T, Santini A, Bottino N, et al. "Awake" extracorporeal membrane oxygenation (ECMO): pathophysiology, technical considerations, and clinical pioneering[J]. Critical Care, 2016, 20(1):150.

17. Murphy, Deirdre A, Hockings, Lisen E, Andrews, Robert K, et al. Extracorporeal Membrane Oxygenation -Hemostatic Complications. Transfusion Medicine Reviews, 2015, 29(2):90-101.

\section{Figures}



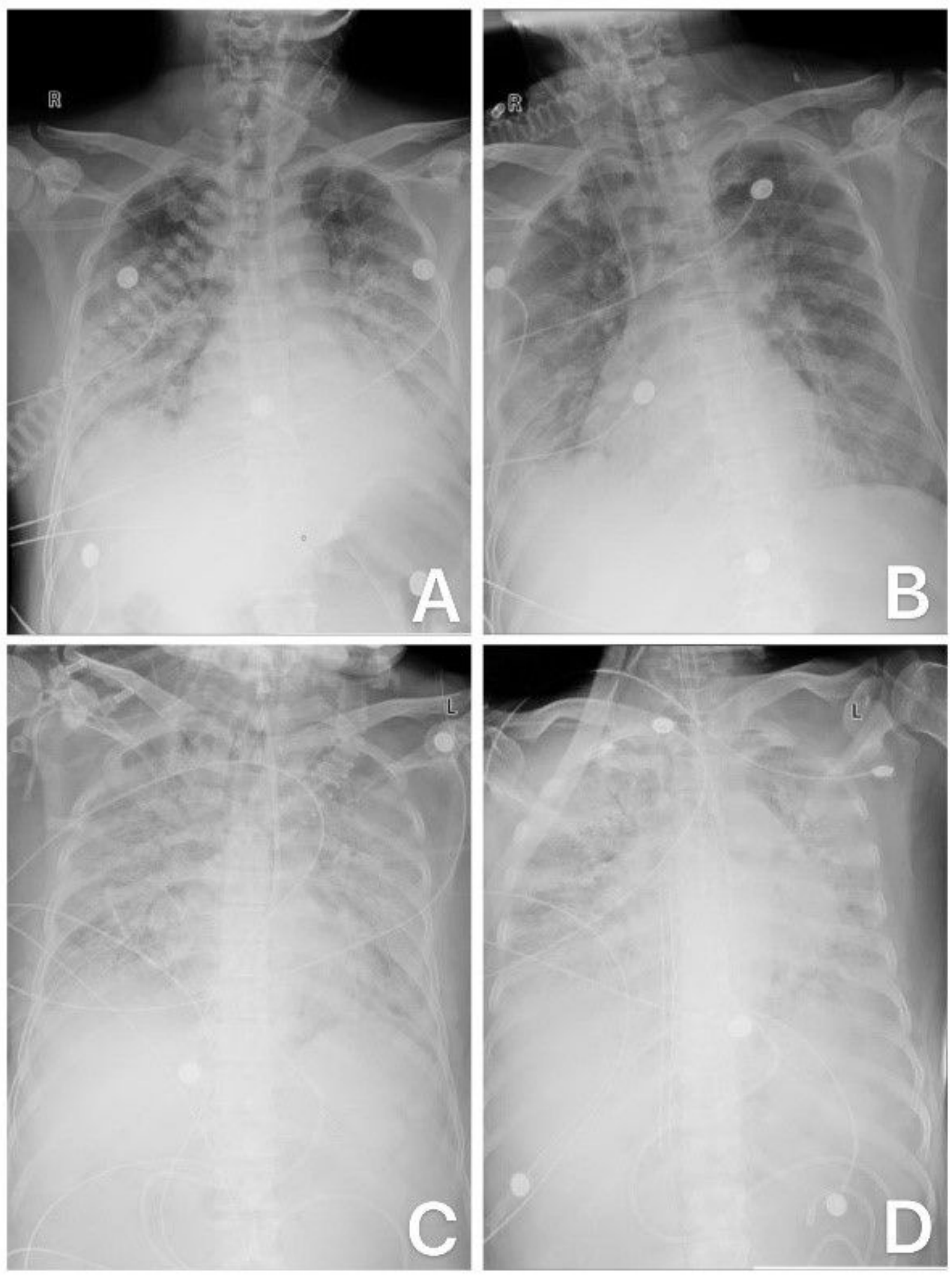

\section{Figure 1}

Chest X-ray images of a 53-year-old man with COVID-19 infection at four time. Notes: A, Chest X-ray at admission; B, Chest $x$ - ray after tracheal intubation; C, Chest $x$ - ray after 96 hours of mechanical ventilation; $\mathrm{D}$, Chest $\mathrm{x}$ - ray after establishing ECMO. 

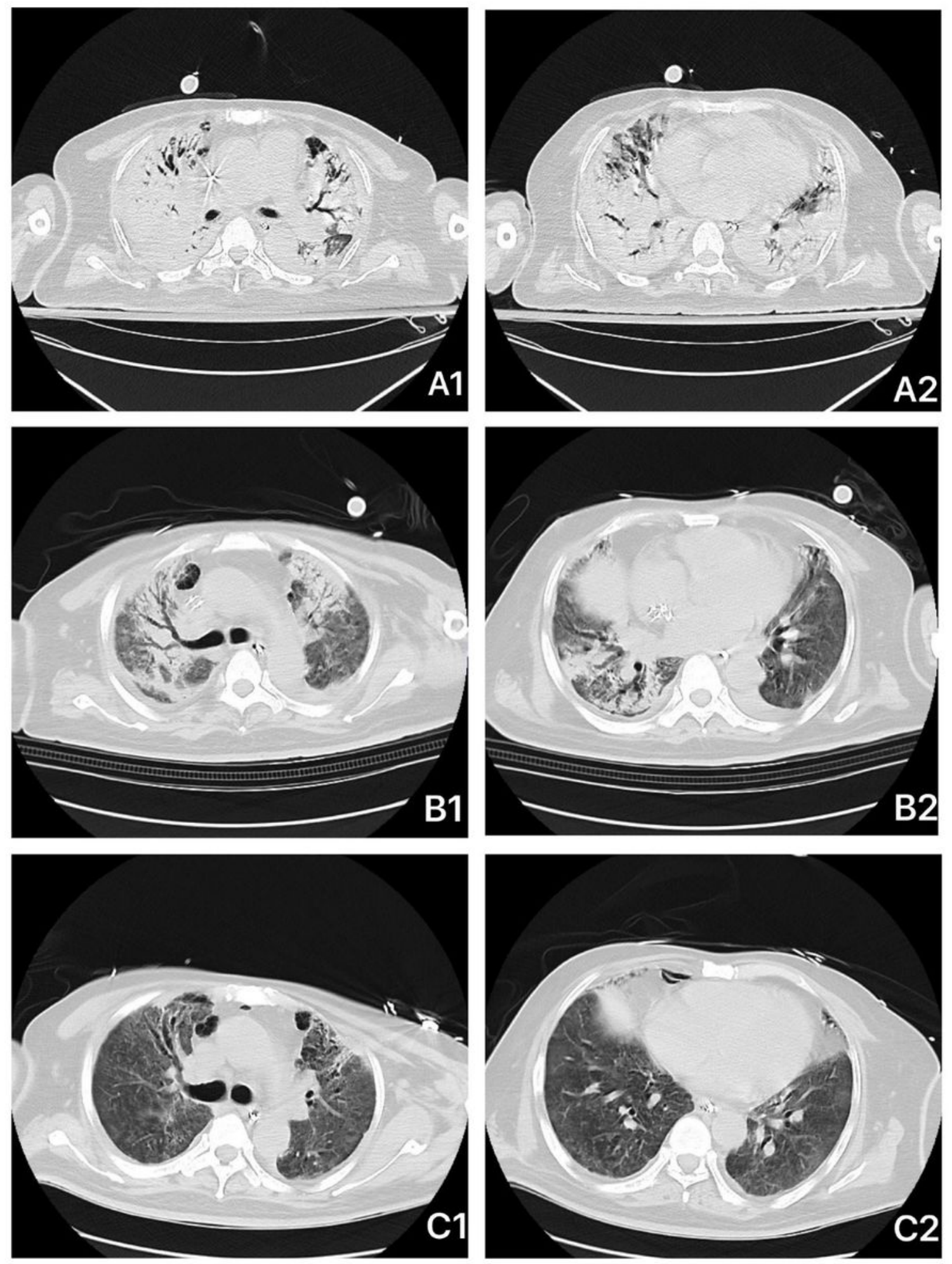

Figure 2

Chest computed tomography (CT) at three time points (A, B, C). Two representative slices of the middle and lower lobe were chosen. Notes: A, 48 hours of extracorporeal membrane oxygenation; B, 1 week of extracorporeal membrane oxygenation and prone position ventilation; $\mathrm{C}, 5$ days of awake ECMO and rehabilitation. 


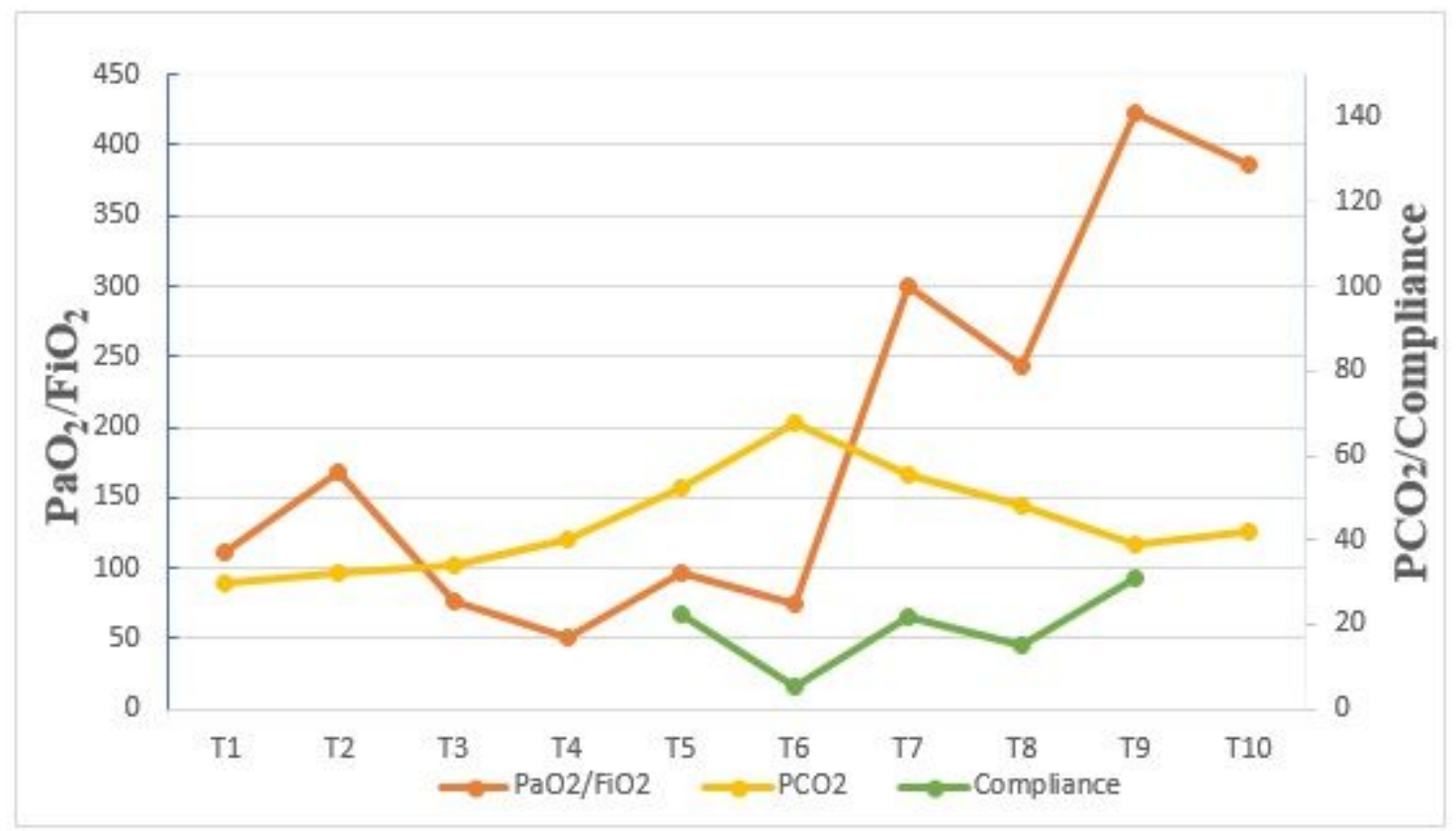

\section{Figure 3}

Changes of PaO2/FiO2 and PCO2/Compliance over time. Abbreviations: T1, Admission; T2, After 24 hours of NIV; T3, ICU admission; T4, Before intubation; T5, After 24 hours of intubation; T6, 24 hours before ECMO establish; T7, Before ECMO weaning off (ECMO oxygen concentration is 21\%邓; T8, 24 hours after ECMO weaning off; T9, Before MV weaning; T10, 24 hours after MV weaning off. 